\title{
Effectively pretraining a speech translation decoder with Machine Translation data
}

\author{
Ashkan Alinejad, Anoop Sarkar \\ Simon Fraser University, Burnaby, BC, Canada \\ \{aalineja,anoop\}@sfu.ca
}

\begin{abstract}
Directly translating from speech to text using an end-to-end approach is still challenging for many language pairs due to insufficient data. Although pretraining the encoder parameters using the $\mathrm{Au}$ tomatic Speech Recognition (ASR) task improves the results in low resource settings, attempting to use pretrained parameters from the Neural Machine Translation (NMT) task has been largely unsuccessful in previous works. In this paper, we will show that by using an adversarial regularizer, we can bring the encoder representations of the ASR and NMT tasks closer even though they are in different modalities, and how this helps us effectively use a pretrained NMT decoder for speech translation.
\end{abstract}

\section{Introduction}

Automatic Speech Translation (AST) aims to directly translate audio signals in the source language into the text words in the target language. For many years, the pipeline of transcribing speech with ASR and then translating with the MT component was a standard method to address the speech translation problem. Having access to lots of data in many language pairs, the cascaded model for speech translation can benefit from well-trained ASR and MT components and generate high-quality translations.

In recent years, it has shown that we can remove the transcription step and build an end-to-end model that is strong enough to compete with the cascaded model (Pino et al., 2019). Such models not only have lower inference latency, but they also do not suffer from the problem of errors that propagate from one component to the next. However, the scarcity of available resources is the main challenge in this task, and a variety of methods are proposed to address this problem. One of the most effective approaches to increase the performance of AST systems is to pretrain the encoder using an ASR model (Bansal et al., 2018). While pretraining the encoder by an ASR model even in different languages shows promising results (Bansal et al., 2019), using a pretrained MT decoder is not beneficial (Berard et al., 2018; Bansal et al., 2018) or slightly improve the result (Sperber et al., 2019) and even in some cases may worsen the results (Bahar et al., 2019).

One explanation for this phenomenon is that the decoder works well only if its input comes from an encoder that it was trained with (Lample et al., 2018). To solve the problem of invariant encoder representations, we make use of an adversarial regularizer in our loss function to bring the output of the ASR encoder closer to the input of MT decoder. We show that this modification can improve the BLEU score by +2.0 BLEU points.

\section{Models}

\subsection{End-to-End Speech Translation}

Similar to conventional MT models, the speech translation task generates translated words in the target language, representing as $\hat{Y}=\left(\hat{y}_{1}, \ldots, \hat{y}_{m}\right)$, given the sequence of source speech features $X=$ $\left(x_{1}, \ldots, x_{n}\right)$. The translation model then minimizes the Cross-Entropy loss $\mathrm{L}_{C E}=\Delta(\hat{Y}, Y)$, where $\Delta$ is the sum of character-level CrossEntropy losses.

We use character-level encoding and decoding using Transformer (Vaswani et al., 2017) as the basic architecture of all our models. For the AST and ASR models, we use similar architecture to (Di Gangi et al., 2019b) with an S-Transformer (Gangi et al., 2019). The main difference between transformer and S-Transformer is the way it encodes the input features. S-Transformer encodes the audio features by passing them into two stacked layers of Convolutional Neural Nets (CNN). Then, 


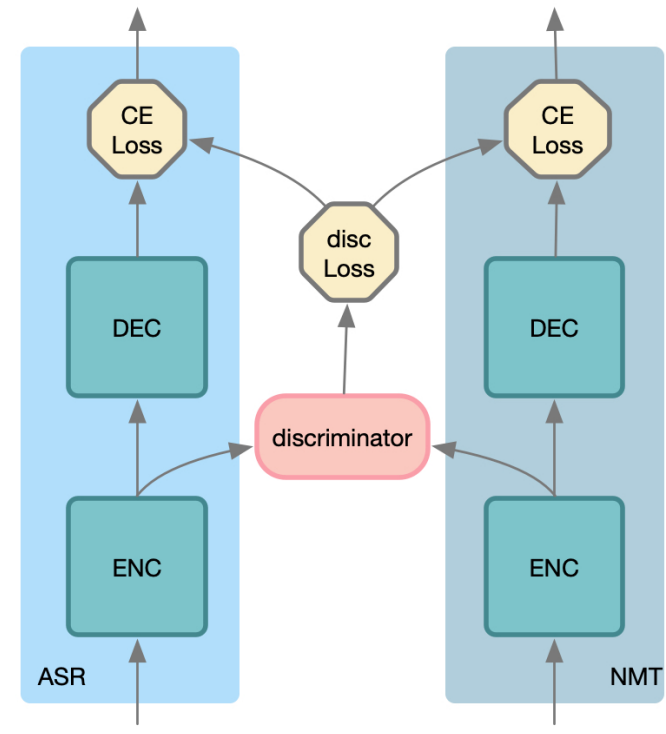

Figure 1: The proposed pretraining method using an adversarial loss.

it uses a 2D self Attention layer to compute the attention matrix using the second CNN's output. We followed the architecture of (Vaswani et al., 2017) in our MT model.

The conventional method for training an AST model is to pretrain ASR and NMT models separately and then transferring parameters of the encoder from ASR and the decoder from MT to the AST model, before starting to train via speech translation data.

\subsection{Aligning encoder representations}

Since we are training the encoder representations of the ASR model and the decoder parameters of the NMT system to work with their own encoder and decoder, pretraining the parameters of the AST model with a speech encoder from ASR and a text decoder from NMT is not ideal. Therefore, we propose to use adversarial training to bring NMT encoder and ASR encoder representations closer together.

An overview of our model is depicted in Figure 1. Instead of separately pretraining the ASR and NMT, we propose to update their parameters simultaneously. In order to add explicit incentives to learn multi-modal representations in the encoder, we will train our NMT and ASR models on both Cross-Entropy loss and a new regularization loss. The final training objective for each task can be formulated as:

$$
\text { Loss }=\mathrm{L}_{C E}+\alpha \mathrm{L}_{D I S C}
$$

where $\mathrm{L}_{C E}$ is the Cross-Entropy loss, $\mathrm{L}_{D I S C}$ is the newly added regularization term, and $\alpha$ is the constant parameter to control the effect of our regularizer. Since $\mathrm{L}_{D I S C}$ is a smaller number compared to $\mathrm{L}_{C E}$, we set $\alpha$ to 5 in all our experiments to make the regularizer loss more perceptible during backward propagation. We are also sharing the parameters of the transformer layers in the encoder between AST and MT models. In the following section, we describe the regularizer.

\subsection{Adversarial regularizer}

Given the embeddings of inputs $x_{i}$ in each modalities (speech features for ASR or character embeddings for NMT), the encoder computes the encoder representations $Z_{x_{i}}$. By passing $Z_{x_{i}}$ to the discriminator, we can train its network by minimizing the loss function $\operatorname{Loss}_{D}=-\mathbb{E}_{\left(x_{i}, m_{i}\right)}\left[\log P_{D}\left(m_{i} \mid Z_{x_{i}}\right)\right]$, where $m_{i}$ is the modality of $x_{i}$, with $m_{i} \in\{$ ASR, NMT $\}$ and $P_{D}$ is the probability of choosing the right modality given the output of encoder.

The encoder of NMT or ASR will be trained in order to deceive the discriminator by minimizing the loss:

$$
\mathrm{L}_{D I S C}=-\mathbb{E}_{\left(x_{i}, m_{i}\right)}\left[\log P_{D}\left(m_{j} \mid Z_{x_{i}}\right)\right]
$$

where $m_{j}=\mathrm{ASR}$ if $m_{i}=\mathrm{NMT}$ and vice versa. By incorporating this regularizer, we ensure that the encoder representations from different modalities (speech and text) become indistinguishable during training.

Our discriminator consists of a three-layer feedforward network with 1024 hidden units, followed by a Leaky-ReLU activation function (Lample et al., 2018).

\section{Experiments}

\subsection{Dataset}

To evaluate our AST systems, we conducted our experiments on two datasets. For the EnglishGerman language pair, we use the MuST-C corpus (Di Gangi et al., 2019a), which consists of 408 hours of speech data aligned with $234 \mathrm{~K}$ translated sentences. For the English-French language pair, we use the full training set of Translation 
Augmented Librispeech (Libri-Trans) corpus (Kocabiyikoglu et al., 2018) with 230 hours of speech aligned with $131 \mathrm{~K}$ french sentences.

We use LibriSpeech corpus (Panayotov et al., 2015) with 960h of English speeches in order to train our ASR system. Since the test and dev sets of Libri-Trans corpus is part of the ASR LibriSpeech dataset, we remove all utterances from ASR LibriSpeech that share the same (chapter-id, readerid) pairs with the test and dev sets in the LibriTrans corpus. For En-De MT training, we use the combination of TED and Opensubtitle2018 corpora ${ }^{12}$ which contains more than $18 \mathrm{M}$ sentences pairs after filtering noisy pairs. The MT training of the English-French language pair uses the En-Fr portion of the WMT14 competition (Bojar et al., 2014).

\subsection{Preprocessing and Evaluation}

For each speech utterance, we extract 40 Melfilterbank energy features with a step size of 10 $\mathrm{ms}$ and a window size of $25 \mathrm{~ms}$. For features extracted from MuSt-C and ASR LibriSpeech, we apply mean and variance normalization for each speaker.

We keep all the texts in our experiments truecase and tokenize them using Moses tokenizer ${ }^{3}$. We remove the punctuation from all English texts (both from the target side of ASR and the source side of MT).

For translation tasks (AST and MT), we report BLEU score (Papineni et al., 2002) on tokenized sentences ${ }^{4}$. We evaluate our ASR systems using Word Error Rate (WER) ${ }^{5}$.

\subsection{Model settings}

For both En-De and En-Fr tasks, we followed the architecture in (Di Gangi et al., 2019b). We use six Transformer layers of size 512 in the encoder and decoder with eight attention heads. The size of feed-forward mechanism is 1024 . The embedding layer in the encoder for the AST task contains two layers of 2D CNNs (Lecun et al., 1998) followed by a ReLU activation function. Each CNN layer has 16 output channels, with a stride of $(2,2)$. We

\footnotetext{
${ }^{1}$ http: / / www . opensubtitles.org/

${ }^{2}$ http://opus.nlpl.eu/ OpenSubtitles-v2018.php

${ }^{3}$ http: / / www. statmt.org/moses/

${ }^{4}$ https://www.nltk.org/_modules/nltk/ translate/bleu_score.html

${ }^{5}$ https://github.com/belambert/ asr-evaluation
}

\begin{tabular}{|c|cc|cc|}
\cline { 2 - 5 } \multicolumn{1}{c|}{} & \multicolumn{2}{c}{ En-De } & \multicolumn{2}{c|}{ En-Fr } \\
\cline { 2 - 5 } \multicolumn{1}{c|}{} & \#param & \#hours & \#param & \#hours \\
\hline $\begin{array}{c}\text { cascaded } \\
\text { NMT }\end{array}$ & $45 \mathrm{M}$ & 27 & $45 \mathrm{M}$ & 13 \\
\hline $\begin{array}{c}\text { cascaded } \\
\text { ASR }\end{array}$ & $31 \mathrm{M}$ & 22.5 & $31 \mathrm{M}$ & 18.2 \\
\hline AST & $31 \mathrm{M}$ & 34 & $31 \mathrm{M}$ & 18 \\
\hline
\end{tabular}

Table 1: The number of parameters and run-time of our models on MuSt-C dataset (En-De) and Libri-Trans dataset (En-Fr).

\begin{tabular}{|lcc|}
\hline Task & En-De & En-Fr \\
\hline \hline cascaded & 18.76 & 15 \\
\hline \hline AST + ASR pre & 18.71 & 14.7 \\
AST + ASR pre + MT pre & 19.05 & 15.3 \\
\hline \hline AST + regularizer & 20.24 & 17.01 \\
\hline
\end{tabular}

Table 2: Results of AST models trained only with AST data. The performance is measured with BLEU score on MuST-C test set.

run all our models on two GeForce GTX 1080 GPUs with 12GB RAM each. The total number of parameters and run-time of our models in Table 1.

\subsection{Training settings}

In all our models, we use the Adam optimizer (Kingma and $\mathrm{Ba}, 2014$ ) with an initial learning rate of 0.00005 . During the first 6000 warm-up updates, we increase it linearly to 0.003 , then decrease it with inverse square root decay (Vaswani et al., 2017). The number of warm-up updates in our MT systems is 8000 .

\section{Results}

In this section, we analyze the effect of our regularizer on two different settings: (A) When we only have access to AST data (section 4.1) and (B) When we can benefit from External data (section 4.2). For each setting, we run experiments on four different models:

1. The cascaded model

2. AST model with pretrained ASR encoder

3. AST model with pretrained ASR encoder and MT decoder

4. Our proposed model with adversarial loss. 


\begin{tabular}{|lcc|}
\hline Task & En-De & En-Fr \\
\hline \hline cascaded & 21.06 & 19.21 \\
\hline \hline AST + ASR pre & 19.01 & 16.13 \\
AST + ASR pre + MT pre & 19.12 & 16.27 \\
\hline \hline AST + regularizer & 20.81 & 17.7 \\
\hline
\end{tabular}

Table 3: BLEU scores of AST models, trained with both AST and external ASR and MT data.

\subsection{Using only AST data}

Table 2 shows the performance of AST models for En-De and En-Fr language pairs. When the cascaded model is restricted to use small AST datasets merely, the model will not be strong enough to beat an AST model with a pretrained encoder and decoder. We should also note that unlike (Bansal et al., 2019; Bahar et al., 2019), where transferring decoder parameters were not effective, in all our AST models, we could only beat the cascaded model by pretraining the decoder.

The last row in the table gives the AST model results, which uses adversarial regularizer during the pretrain step. As we can see, training the NMT and the ASR models simultaneously can help pretrained components be compatible with each other and improve the final performance by 1.2 and 1.7 BLEU scores for En-De and En-Fr language pairs respectively.

\subsection{Using both AST and External data}

Limiting the training data for the speech translation models to AST datasets is not a realistic assumption for many language pairs, and in practice, the cascaded model can greatly benefit from the large amounts of NMT and ASR corpora.

Table 3 summarizes the effects of adding external training data to our experiments. Adding external data can boost the performance of the cascaded model and by comparing Table 2 and 3, we can see that the additional NMT and ASR data can improve the translation quality of the cascaded model by +2 BLEU scores, while it can barely affect the AST model with pretrained encoder and the decoder. Consequently, the gap between the AST model and the cascaded system increases by around +3 BLEU scores for En-Fr and +2 BLEU scores for the En-De language pair.

As we can see in the last row of Table 3, adding our proposed pretraining step can help the model perform better during training, and compared to the conventional pretraining step, we can see an in- crease of more than 1 BLEU point in each language pair. Although the cascaded model by having access to all the pretrained parameters (the encoder and decoder of both NMT and ASR) still has better translation quality, we can bring the performance of an end-to-end model closer to it by adding the new regularizer. It is also important to note that since we are not changing the final structure of the AST model, most of the other techniques for further improving the translation quality, such as data augmentation, which was examined in previous studies (McCarthy et al., 2020; Park et al., 2019) can also be applied. But we won't study them in this paper.

\section{Related Work}

The cascaded pipeline of transcribing speech signals and then translating them using an MT component (Ney, 1999; Cho et al., 2017) was for many years the standard design of speech translation systems (Inaguma et al., 2019). The idea of having an end-to-end structure for this task showed promising results in the works of (Adams et al., 2016; Duong et al., 2016; Bérard et al., 2016; Anastasopoulos et al., 2016; Anastasopoulos and Chiang, 2017; Bansal et al., 2017). After the success of (Weiss et al., 2017) in creating a powerful model for ST systems, more recent studies focused on exploring their power, and one of the main approaches to boost the performance of such models is to make use of available data from other tasks, such as ASR and NMT. (Weiss et al., 2017; Anastasopoulos and Chiang, 2018; Sperber et al., 2019) show that multitask learning can be effective and (Jia et al., 2019; Pino et al., 2019; Park et al., 2019; McCarthy et al., 2020) investigate various data augmentation techniques. The impact of pretraining the encoder with ASR model is also studied in (Berard et al., 2018; Bansal et al., 2018, 2019). In experiments of (Bahar et al., 2019; Bansal et al., 2019) the performance gain of pretraining the decoder with an MT model was marginal.

(Kano et al., 2020) addresses the ASR encoder and MT decoder gap problem by proposing a "Transcoder" and use smooth-L1 loss to bring ASR hidden representation close to MT encoder hidden representation.

The idea of modifying loss function in AST models was also discussed in (Sperber et al., 2019). Their formulation of the additional loss is different from ours, and they use their additional loss 
function in a different NMT architecture from ours.

The idea of adding adversarial regularizer was discussed in other tasks such as unsupervised MT (Lample et al., 2018) or zero-shot translation (Pham et al., 2019). The closest research to our work is (Arivazhagan et al., 2019), which uses a similar adversarial network to bring encoder representations closer together. However, they apply their model to the zero-shot machine translation task, with a different architecture. They also apply their regularizer to the representations of the different languages with the same modalities.

\section{Conclusion}

In this paper, we study the impact of pretraining an AST decoder using an MT model and propose a method to make the pretraining step more effective. We show that we can align the latent representations of different modalities by using adversarial loss and make the ASR encoder more compatible with the MT decoder. Our experiments demonstrate that we can improve the performance by around 1.5 BLEU points on two language pairs compared to conventional pretraining methods.

\section{Acknowledgments}

We would like to thank the anonymous reviewers for their helpful comments. The research was partially supported by the Natural Sciences and Engineering Research Council of Canada grants NSERC RGPIN-2018-06437 and RGPAS-2018522574 and a Department of National Defence (DND) and NSERC grant DGDND-2018-00025.

\section{References}

Oliver Adams, Graham Neubig, Trevor Cohn, Steven Bird, Quoc Truong Do, and Satoshi Nakamura. 2016. Learning a lexicon and translation model from phoneme lattices. In Proceedings of the 2016 Conference on Empirical Methods in Natural Language Processing, pages 2377-2382, Austin, Texas. Association for Computational Linguistics.

Antonios Anastasopoulos and David Chiang. 2017. A case study on using speech-to-translation alignments for language documentation. In Proceedings of the 2nd Workshop on the Use of Computational Methods in the Study of Endangered Languages, pages 170-178, Honolulu. Association for Computational Linguistics.

Antonios Anastasopoulos and David Chiang. 2018. Tied multitask learning for neural speech translation. In Proceedings of the 2018 Conference of the North
American Chapter of the Association for Computational Linguistics: Human Language Technologies, Volume 1 (Long Papers), pages 82-91, New Orleans, Louisiana. Association for Computational Linguistics.

Antonios Anastasopoulos, David Chiang, and Long Duong. 2016. An unsupervised probability model for speech-to-translation alignment of low-resource languages. In Proceedings of the 2016 Conference on Empirical Methods in Natural Language Processing, pages 1255-1263, Austin, Texas. Association for Computational Linguistics.

Naveen Arivazhagan, Ankur Bapna, Orhan Firat, Roee Aharoni, Melvin Johnson, and Wolfgang Macherey. 2019. The missing ingredient in zero-shot neural machine translation. ArXiv, abs/1903.07091.

Parnia Bahar, Tobias Bieschke, and Hermann Ney. 2019. A comparative study on end-to-end speech to text translation. 2019 IEEE Automatic Speech Recognition and Understanding Workshop (ASRU), pages 792-799.

Sameer Bansal, Herman Kamper, Karen Livescu, Adam Lopez, and Sharon Goldwater. 2018. Low-resource speech-to-text translation. CoRR, abs/1803.09164.

Sameer Bansal, Herman Kamper, Karen Livescu, Adam Lopez, and Sharon Goldwater. 2019. Pretraining on high-resource speech recognition improves low-resource speech-to-text translation. In Proceedings of the 2019 Conference of the North American Chapter of the Association for Computational Linguistics: Human Language Technologies, NAACL-HLT 2019, Minneapolis, MN, USA, June 27, 2019, Volume 1 (Long and Short Papers), pages 58-68. Association for Computational Linguistics.

Sameer Bansal, Herman Kamper, Adam Lopez, and Sharon Goldwater. 2017. Towards speech-to-text translation without speech recognition. CoRR, abs/1702.03856.

Alexandre Berard, Laurent Besacier, Ali Kocabiyikoglu, and Olivier Pietquin. 2018. End-to-end automatic speech translation of audiobooks. pages 6224-6228.

Alexandre Bérard, Olivier Pietquin, Laurent Besacier, and Christophe Servan. 2016. Listen and Translate: A Proof of Concept for End-to-End Speech-to-Text Translation. In NIPS Workshop on end-to-end learning for speech and audio processing, Barcelona, Spain.

Ondřej Bojar, Christian Buck, Christian Federmann, Barry Haddow, Philipp Koehn, Johannes Leveling, Christof Monz, Pavel Pecina, Matt Post, Herve Saint-Amand, Radu Soricut, Lucia Specia, and Aleš Tamchyna. 2014. Findings of the 2014 workshop on statistical machine translation. In Proceedings of the Ninth Workshop on Statistical Machine Translation, 
pages $12-58$, Baltimore, Maryland, USA. Association for Computational Linguistics.

Eunah Cho, Jan Niehues, and Alex Waibel. 2017. Nmtbased segmentation and punctuation insertion for real-time spoken language translation. In Proc. Interspeech 2017, pages 2645-2649.

Mattia A. Di Gangi, Roldano Cattoni, Luisa Bentivogli, Matteo Negri, and Marco Turchi. 2019a. MuST-C: a Multilingual Speech Translation Corpus. In Proceedings of the 2019 Conference of the North American Chapter of the Association for Computational Linguistics: Human Language Technologies, Volume 1 (Long and Short Papers), pages 2012-2017, Minneapolis, Minnesota. Association for Computational Linguistics.

Mattia A. Di Gangi, Matteo Negri, Viet Nhat Nguyen, Amirhossein Tebbifakhr, and Marco Turchi. 2019b. Data augmentation for end-to-end speech translation: Fbk@iwslt'19.

Long Duong, Antonios Anastasopoulos, David Chiang, Steven Bird, and Trevor Cohn. 2016. An attentional model for speech translation without transcription. In Proceedings of the 2016 Conference of the North American Chapter of the Association for Computational Linguistics: Human Language Technologies, pages 949-959, San Diego, California. Association for Computational Linguistics.

Mattia A. Di Gangi, Matteo Negri, and Marco Turchi. 2019. Adapting Transformer to End-to-End Spoken Language Translation. In Proc. Interspeech 2019, pages 1133-1137.

Hirofumi Inaguma, Kevin Duh, Tatsuya Kawahara, and Shinji Watanabe. 2019. Multilingual end-to-end speech translation. 2019 IEEE Automatic Speech Recognition and Understanding Workshop (ASRU), pages 570-577.

Ye Jia, Melvin Johnson, Wolfgang Macherey, Ron Weiss, Yuan Cao, Chung-Cheng Chiu, Naveen Ari, Stella Laurenzo, and Yonghui Wu. 2019. Leveraging weakly supervised data to improve end-to-end speech-to-text translation. pages 7180-7184.

Takatomo Kano, Sakriani Sakti, and Satoshi Nakamura. 2020. End-to-end speech translation with transcoding by multi-task learning for distant language pairs. IEEE/ACM Trans. Audio, Speech and Lang. Proc., 28:1342-1355.

Diederik Kingma and Jimmy Ba. 2014. Adam: A method for stochastic optimization. International Conference on Learning Representations.

Ali Can Kocabiyikoglu, Laurent Besacier, and Olivier Kraif. 2018. Augmenting librispeech with French translations: A multimodal corpus for direct speech translation evaluation. In Proceedings of the Eleventh International Conference on Language Resources and Evaluation (LREC 2018), Miyazaki, Japan. European Language Resources Association (ELRA).
Guillaume Lample, Alexis Conneau, Ludovic Denoyer, and Marc' Aurelio Ranzato. 2018. Unsupervised machine translation using monolingual corpora only. In International Conference on Learning Representations.

Y. Lecun, L. Bottou, Y. Bengio, and P. Haffner. 1998. Gradient-based learning applied to document recognition. Proceedings of the IEEE, 86(11):2278-2324.

A. D. McCarthy, L. Puzon, and J. Pino. 2020. Skinaugment: Auto-encoding speaker conversions for automatic speech translation. In ICASSP 2020 - 2020 IEEE International Conference on Acoustics, Speech and Signal Processing (ICASSP), pages 7924-7928.

H. Ney. 1999. Speech translation: coupling of recognition and translation. In 1999 IEEE International Conference on Acoustics, Speech, and Signal Processing. Proceedings. ICASSP99 (Cat. No.99CH36258), volume 1, pages 517-520 vol.1.

V. Panayotov, G. Chen, D. Povey, and S. Khudanpur. 2015. Librispeech: An asr corpus based on public domain audio books. In 2015 IEEE International Conference on Acoustics, Speech and Signal Processing (ICASSP), pages 5206-5210.

Kishore Papineni, Salim Roukos, Todd Ward, and WeiJing Zhu. 2002. Bleu: a method for automatic evaluation of machine translation. In Proceedings of the 40th Annual Meeting of the Association for Computational Linguistics, pages 311-318, Philadelphia, Pennsylvania, USA. Association for Computational Linguistics.

Daniel S. Park, William Chan, Yu Zhang, ChungCheng Chiu, Barret Zoph, Ekin D. Cubuk, and Quoc V. Le. 2019. Specaugment: A simple data augmentation method for automatic speech recognition. In INTERSPEECH.

Ngoc-Quan Pham, Jan Niehues, Thanh-Le Ha, and Alex Waibel. 2019. Improving zero-shot translation with language-independent constraints. In Proceedings of the Forth Conference on Statistical Machine Translation (WMT 2019).

Juan Pino, Liezl Puzon, Jiatao Gu, Xutai Ma, Arya D. McCarthy, and Deepak Gopinath. 2019. Harnessing indirect training data for end-to-end automatic speech translation: Tricks of the trade.

Matthias Sperber, Graham Neubig, Jan Niehues, and Alex Waibel. 2019. Attention-passing models for robust and data-efficient end-to-end speech translation. Transactions of the Association for Computational Linguistics, 7:313-325.

Ashish Vaswani, Noam Shazeer, Niki Parmar, Jakob Uszkoreit, Llion Jones, Aidan N Gomez, Ł ukasz Kaiser, and Illia Polosukhin. 2017. Attention is all you need. In I. Guyon, U. V. Luxburg, S. Bengio, 
H. Wallach, R. Fergus, S. Vishwanathan, and R. Garnett, editors, Advances in Neural Information Processing Systems 30, pages 5998-6008. Curran Associates, Inc.

Ron J. Weiss, Jan Chorowski, Navdeep Jaitly, Yonghui Wu, and Zhifeng Chen. 2017. Sequence-tosequence models can directly translate foreign speech. In INTERSPEECH. 